\title{
IgG Seroconversion and Pathophysiology in Severe Acute Respiratory Syndrome Coronavirus 2 Infection
}

Henry M. Staines, ${ }^{1}$ Daniela E. Kirwan, ${ }^{1}$ David J. Clark, ${ }^{1}$ Emily R. Adams, Yolanda Augustin, Rachel L. Byrne, Michael Cocozza, Ana I. Cubas-Atienzar, Luis E. Cuevas, Martina Cusinato, Benedict M.O. Davies,

Mark Davis, Paul Davis, Annelyse Duvoix, Nicholas M. Eckersley, Daniel Forton, Alice J. Fraser, Gala Garrod, Linda Hadcocks, Qinxue Hu, Michael Johnson, Grant A. Kay, Kesja Klekotko, Zawditu Lewis, Derek C. Macallan, Josephine Mensah-Kane, Stefanie Menzies, Irene Monahan, Catherine M. Moore,

Gerhard Nebe-von-Caron, Sophie I. Owen, Chris Sainter, Amadou A. Sall, James Schouten, Christopher T. Williams, John Wilkins, Kevin Woolston, Joseph R.A. Fitchett, Sanjeev Krishna, ${ }^{2}$ Tim Planche ${ }^{2}$

We investigated the dynamics of seroconversion in severe acute respiratory syndrome coronavirus 2 (SARS-CoV-2) infection. During March 29-May 22, 2020, we collected serum samples and associated clinical data from 177 persons in London, UK, who had SARS-CoV-2 infection. We measured IgG against SARS-CoV-2 and compared antibody levels with patient outcomes, demographic information, and laboratory characteristics. We found that $2.0 \%-8.5 \%$ of persons did not seroconvert 3-6 weeks after infection. Persons who seroconverted were older, were more likely to have concurrent conditions, and had higher levels of inflammatory markers. Non-White persons had higher antibody concentrations than those who identified as White; these concentrations did not decline during follow-up. Serologic assay results correlated with disease outcome, race, and other risk factors for severe SARS-CoV-2 infection. Serologic assays can be used in surveillance to clarify the duration and protective nature of humoral responses to SARS-CoV-2 infection.

Severe acute respiratory syndrome coronavirus $\mathrm{S}_{2}$ (SARS-CoV-2) is a betacoronavirus that causes coronavirus disease (COVID-19), a respiratory infection with systemic involvement and an estimated 1\% death rate (1). COVID-19 was first documented in Wuhan, China (2), at the end of 2019. The outbreak quickly transformed into a pandemic. Countries have tried to manage the pandemic by implementing different strategic interventions with varying levels of success (3). Experts agree that diagnostic tests, and the subsequent interventions they generate, are essential to controlling SARS-CoV-2 transmission.

Reverse transcription PCR (RT-PCR) relies on RNA sequencing rather than viral proteins, enabling researchers to develop assays shortly after the viral sequence is identified. Because of this advantage, RTPCR quickly became a common testing method for COVID-19 (4). However, the urgent global scale-up of nucleic acid amplification testing, including PCR, exposed supply chain vulnerabilities, such as shortages of swabs and reagents. Diagnostic tests remain unaffordable in many developing countries, limiting national containment strategies.

Serologic assays for viral infections can contribute to vaccine development, diagnostic deployment, and prescription of new therapeutics. They
Author affiliations: St. George's, University of London, London, UK (H.M. Staines, D.E. Kirwan, D.J. Clark, Y. Augustin, M. Cuisinato, B.M.O. Davies, N.M. Eckersley, D. Forton, L. Hadcocks, Q. Hu, D.C. Macallan, I. Monahan, C.M. Moore, S. Krishna, T. Planche); Liverpool School of Tropical Medicine, Liverpool, UK (E.R. Adams, R.L. Byrne, A.I. Cubas-Atienzar, L.E. Cuevas, A.J. Fraser, G. Garrod, G.A. Kay, S. Menzies, S.I. Owen, C.T. Williams); Mologic, Thurleigh, UK (M. Cocozza, M. Davis, P. Davis, A. Duvoix, M. Johnson, K. Klekotko, Z. Lewis, J. Mensah-Kane, G. Nebe-von-Caron, C. Sainter, J. Schouten, J. Wilkins,
K. Woolston, J.R.A. Fitchett); St. George's University Hospitals National Health Services Foundation Trust, London (D. Forton, S. Krishna, T. Planche); Institut Pasteur, Dakar, Senegal (A.A. Sall); Universitätsklinikum Tübingen, Tübingen, Germany (S. Krishna); Centre de Recherches Médicales de Lambaréné, Lambaréné, Gabon (S. Krishna)

DOI: https://doi.org/10.3201/eid2701.203074

${ }^{1}$ These first authors contributed equally to this article.

${ }^{2}$ These senior authors contributed equally to this article. 
might also offer insight into pathophysiological aspects of COVID-19. We used ELISA (Mologic Ltd., https:/ / mologic.co.uk) for COVID-19 to characterize the serologic response in SARS-CoV-2 infection. These tests were designed for affordability and accuracy, enabling access and manufacture in lowand middle-income countries. We used these tests on serum samples from persons with confirmed SARS-CoV-2 infection in London, UK, to identify demographic and clinical variables that might influence antibody responses.

\section{Methods}

\section{Ethics}

Development of the SARS-CoV-2 IgG ELISA is available elsewhere (E.R. Adams, unpub. data, https:// www.medrxiv.org/content/10.1101/2020. 04.29.20082099v1). We analyzed antibody dynamics using anonymized excess diagnostic material from patients with PCR-confirmed SARS-CoV-2 infection. The study was sponsored by St. George's Hospital National Health Services Foundation Trust (London) and has Institutional Review Board ethics approval (Development and Assessment of Rapid Testing for SARS-CoV-2 outbreak study; Integrated Research Application System project ID: 282104; Research Ethics Committee reference: 20/ SC/0171). The trial is registered at ClinicalTrials. gov under NCT04351646.

\section{Reference RT-PCR}

Staff at St. George's Hospital used Sigma Virocult (MWE, https:/ / www.mwe.co.uk) to collect nose and throat swab samples from patients with SARS-CoV-2 infection; we prepared the samples with RNA extraction kits (Roche Molecular Systems Inc., https:/ / www. lifescience.roche.com). We confirmed infection with the RealStar SARS-CoV-2 RT-PCR Kit selective for the $\mathrm{S}$ and E genes (Altona Diagnostics GmbH, https:// www.altona-diagnostics.com) or cobas SARS-CoV-2 Test selective for the E gene and open reading frames $1 \mathrm{ab}$ (Roche Molecular Systems, Inc.).

\section{Clinical Samples}

South West London Pathology (London) provides microbial diagnostic testing for the region, including St. George's Hospital, a tertiary teaching hospital. We obtained excess diagnostic material from South West London Pathology in the form of serum samples from patients with RT-PCR confirmed SARS$\mathrm{CoV}-2$ infection. The serum samples were anonymized and stored at $4^{\circ} \mathrm{C}$ for $\leq 2$ weeks. Patients were sampled longitudinally to assess antibody dynamics; the data comprised $\geq 30$ samples per day. If samples became unavailable from 1 patient (i.e., the patient was discharged or died), we added a new patient to the cohort. Excess diagnostic material was collected from 177 persons during March 29-May 22, 2020. The study population consisted of $9.9 \%(177 / 1,785)$ of persons (patients and staff) who tested positive for SARS-CoV-2 infection at South West London Pathology during this period.

\section{Participants and Clinical Data}

We obtained data from patients' electronic medical records. We coded outcomes (as of May 22) as hospital admission, intensive care unit stay, death, or discharge. We recorded the length of hospital stay of patients who were discharged or died. We considered peaks of inflammatory markers (e.g., C-reactive protein [CRP]) to be the highest values recorded from 5 days before the first positive swab sample through the end of the study. We obtained blood values at the time of diagnosis (within 3 days after the first positive swab sample was taken).

\section{ELISA for SARS-CoV-2 IgG}

Weused the COVID-19IgGELISA developed by Mologic Ltd. and manufactured by Omega (Omega Diagnostics Group PLC, http:/ / www.omega-diagnostics.com), according to the manufacturer's instructions (Appendix 1, https://wwwnc.cdc.gov/EID/article/27/1/20-3074App1.pdf). The assay contained the spike and nucleoprotein antigens of SARS-CoV-2. Between plate coefficients of variation were $21.0 \%$ (lower cutoff) and $16.5 \%$ (positive control; $\mathrm{n}=16$ ). Higher ambient temperatures in the laboratory resulted in higher optical density readings (Appendix 2, https://wwwnc.cdc.gov/EID/ article/27/1/20-3074- App2.xlsx).

\section{Statistical Analyses}

We cross-checked and normalized raw ELISA data to enable comparison (Appendix 1; Figure 1). We also resolved manual handling errors (Appendix 1). We applied 2-tailed parametric and nonparametric tests as appropriate, using PRISM version 8.0 (https:// www.graphpad.com) for data analysis and display. We conducted a 1-way analysis of variance to compare the effects of race and demographic information on patient outcomes. We used multivariate linear regression to determine the relationship between mean normalized optical density (NOD) and age, sex, peak $\mathrm{CRP}$, number of concurrent conditions, respiratory symptoms, and race. 


\section{Patient and Public Involvement}

We acknowledge the importance of patient and public involvement in clinical studies. However, because of the rapid progression of COVID-19 and the challenges of lockdown in the United Kingdom, we did not have sufficient time to involve patients and members of the public in the development, implementation, or interpretation of this study.

\section{Results}

We studied 177 patients who provided 645 distinct excess diagnostic material samples (Table 1). Patients were from diverse ethnic backgrounds $(34 \%$ White, 35\% non-White, 31\% unreported; Appendix 1 Table 1), the a median age was 64 years (interquartile range [IQR] 52-77 years). Fifty-seven percent were male, and $73 \%$ had $\geq 1$ concurrent

\begin{tabular}{|c|c|}
\hline Characteristics & Results \\
\hline Median age, y (IQR) & $64(52-77)$ \\
\hline Median body mass index (IQR)† & $25.4(21.9-30.5)$ \\
\hline \multicolumn{2}{|l|}{ Sex } \\
\hline M & $100(56.5)$ \\
\hline $\mathrm{F}$ & $77(43.5)$ \\
\hline \multicolumn{2}{|l|}{ Race } \\
\hline White & $60(33.9)$ \\
\hline Non-White & $61(34.5)$ \\
\hline Other/not known & $56(31.6)$ \\
\hline \multicolumn{2}{|l|}{ Concurrent conditions } \\
\hline 0 & $47(26.6)$ \\
\hline 1 & $52(29.4)$ \\
\hline 2 & $50(28.2)$ \\
\hline$\geq 3$ & $28(15.8)$ \\
\hline \multicolumn{2}{|l|}{ Symptoms } \\
\hline Symptomatic & $143(80.8)$ \\
\hline $\begin{array}{l}\text { Median days from symptom onset to } \\
\text { PCR (IQR) }\end{array}$ & $6(3-9)$ \\
\hline \multicolumn{2}{|l|}{ Diagnostic site } \\
\hline Emergency department & $90(50.8)$ \\
\hline Outpatient & $12(6.8)$ \\
\hline Ward & $54(30.5)$ \\
\hline Intensive care unit & $14(7.9)$ \\
\hline Occupational health staff & $7(4.0)$ \\
\hline \multicolumn{2}{|l|}{ Treatment location } \\
\hline Occupational health staff & $7(4.0)$ \\
\hline Outpatient & $4(2.3)$ \\
\hline Hospital & $166(93.8)$ \\
\hline Admitted to intensive care unit & $63(38.0)$ \\
\hline \multicolumn{2}{|l|}{ Outcomes } \\
\hline Never hospitalized & $11(6.2)$ \\
\hline Discharged & $108(61.0)$ \\
\hline Median length of stay, $d$ (IQR) & $19.3(10.6-31.1)$ \\
\hline Death & $44(24.9)$ \\
\hline Median length of stay, $d(I Q R)$ & $19.1(14.8-24.8)$ \\
\hline Death and/or ICU admission & $80(45.2)$ \\
\hline Still in hospitalł & $14(7.9)$ \\
\hline $\begin{array}{l}{ }^{*} \text { Values are no. (\%) except as indicated. ICU } \\
\text { interquartile range. } \\
\text { †Height unavailable for } 13 \text { patients. } \\
\ddagger \text { As of May 22, } 2020 \text {. }\end{array}$ & care unit; IQR, \\
\hline
\end{tabular}

condition. Nineteen percent were asymptomatic and did not report respiratory symptoms at admission; these patients tested positive for SARS-CoV-2 infection while receiving treatment for other conditions. Among the 143 symptomatic patients, the median time from symptom onset to testing was 6 days (IQR 3-9 days). Of the 177 patients, 166 (94\%) were hospitalized, $7(4 \%)$ were staff, and $4(2 \%)$ were outpatients. Of the hospitalized patients, $44(27 \%)$ died (median time to death was 19.1 days [IQR 14.8-24.8 days]), 108 (65\%) were discharged (median length of stay was 19.3 days [IQR 10.6-31.1 days]), and 14 $(8 \%)$ remained hospitalized at the end of the study. Sixty-three $(38 \%)$ patients were admitted to intensive care during the study.

We normalized optical densities proportional to levels of SARS-CoV-2 IgG (Figure 1, panel A). Of the 177 patients, 149 (84\% [95\% CI 78\%-89\%]) had already seroconverted at the time of the first serologic test, 13 (7.3\% [95\% CI 4.3\%-12.1\%]) seroconverted after the first serologic test, and 15 (8.5 \% [95\% CI 5.2-13.5\%]) did not seroconvert during the entire follow-up period (Appendix 1 Figure 2). Of the 15 patients who did not seroconvert, samples from beyond day 20 were available for 4 patients (26\%); we did not detect IgG in these samples. This finding suggests that 2.0\%-8.5\% of patients might not develop detectable IgG against SARS-CoV-2.

We plotted NODs by time after a patient's first positive swab sample (Figure 1, panel B) and after symptom onset (Figure 1, panel C). NODs plateaued $\approx 12$ days after PCR and $\approx 19$ days after symptom onset; this time difference is consistent with the median time of 6 days between symptom onset and PCR. After seroconversion, mean NODs remained stable over the course of the study (up to $\approx 60$ days after symptom onset).

We assessed whether the rate of seroconversion was associated with patient age ( $\leq 70$ or $>70$ years), sex, or respiratory symptoms. None of these variables were discernably associated with seroconversion rates (Appendix 1 Figure 3, panels A-C). NOD IgG levels were not associated with sex or the presence of respiratory symptoms. (Appendix 1 Figure 4, panels A, B).

Patients of non-White race had higher mean NODs than those of White race (1.06 vs. $0.85 ; F=1.61$, $\mathrm{df}=119 ; \mathrm{p}=0.04$ by unpaired Student $t$-test) (Appendix 1 Figure 4, panel C). No other differences were associated with race. We used a multivariate analysis to identify variables independently associated with NODs; the mean NOD was associated only with age, peak CRP, and race. Although age, sex, peak $\mathrm{CRP}$, number of concurrent conditions, respiratory 
symptoms, and race were associated with patient outcome in the univariate analysis, only peak CRP was associated with poor outcome in the multivariate analysis (Appendix 1).

Persons who seroconverted were older than those who did not (median age 65.5 vs. 41.0 years; $\mathrm{p}<0.01$ by Mann-Whitney test) and more likely to have $\geq 1$ concurrent condition (124/130 vs. 38/47; $p<0.01$ by Fisher exact test). History of hypertension was associated with a higher probability of seroconversion (74/75 persons with hypertension vs. 88/102 persons without hypertension; $\mathrm{p}<0.01$ by Fisher exact test). Body mass index was higher among the group who seroconverted ( 25.7 vs. $21.2 ; p=0.03$ by MannWhitney test).

Unlike other markers of inflammation, CRP is routinely measured in patients with COVID-19. Rising CRP levels are indicators of a poor prognosis (if other causes are excluded), and are associated with cytokine release syndrome (5; Y. Woo, unpub. data, https:/ / osf. io/mxsvw). CRP levels were significantly higher in patients with respiratory symptoms at diagnosis than in those without symptoms (Figure 2, panel A). Patients who died or required intensive care during the study period had higher CRP levels than patients who did not die or require intensive care (Figure 2, panel B). Patients who did not seroconvert had lower CRP levels than those who did (Figure 2, panel C). Peak CRP levels had more pronounced associations with outcomes and seroconversion than did CRP levels at the time of the first positive swab sample result (Figure 2, panels D-F). CRP levels peaked a median of 12 days (IQR 8-17 days) after symptom onset and 4 days (IQR 1-11 days) after the first positive PCR result. Other inflammatory markers, such as peak D-dimer, fibrinogen, and ferritin, were also higher in patients with respiratory symptoms at diagnosis. However, these data were available for fewer patients (Table 2; Appendix 1 Table 3).

\section{Discussion}

Our results illustrate serologic responses over the course of SARS-CoV-2 infection. Serologic tests can enhance diagnostic capability, especially during later infection $(4,6,7)$, when viral loads might decrease. Serologic testing might also inform surveillance, seroepidemiologic studies, and contact tracing. Our study shows that a substantial proportion of COVID-19 patients require 3-6 weeks to generate antibodies. Furthermore, $2.0 \%-8.5 \%$ of patients do not have detectable antibodies within 60 days after infection. Most research on antibody dynamics came from China during the early stages of the pandemic $(4,6,8)$. Here, we describe variables that influence IgG dynamics in
SARS-CoV-2 infections in diverse populations.

The performance metrics of this ELISA (Figure 1; Appendix 2) are comparable to other validated assays. This first-generation ELISA might confirm infection in patients without a virologic diagnosis. We applied this test to study an ethnically and clinically diverse population. In most persons who seroconverted, the conversion
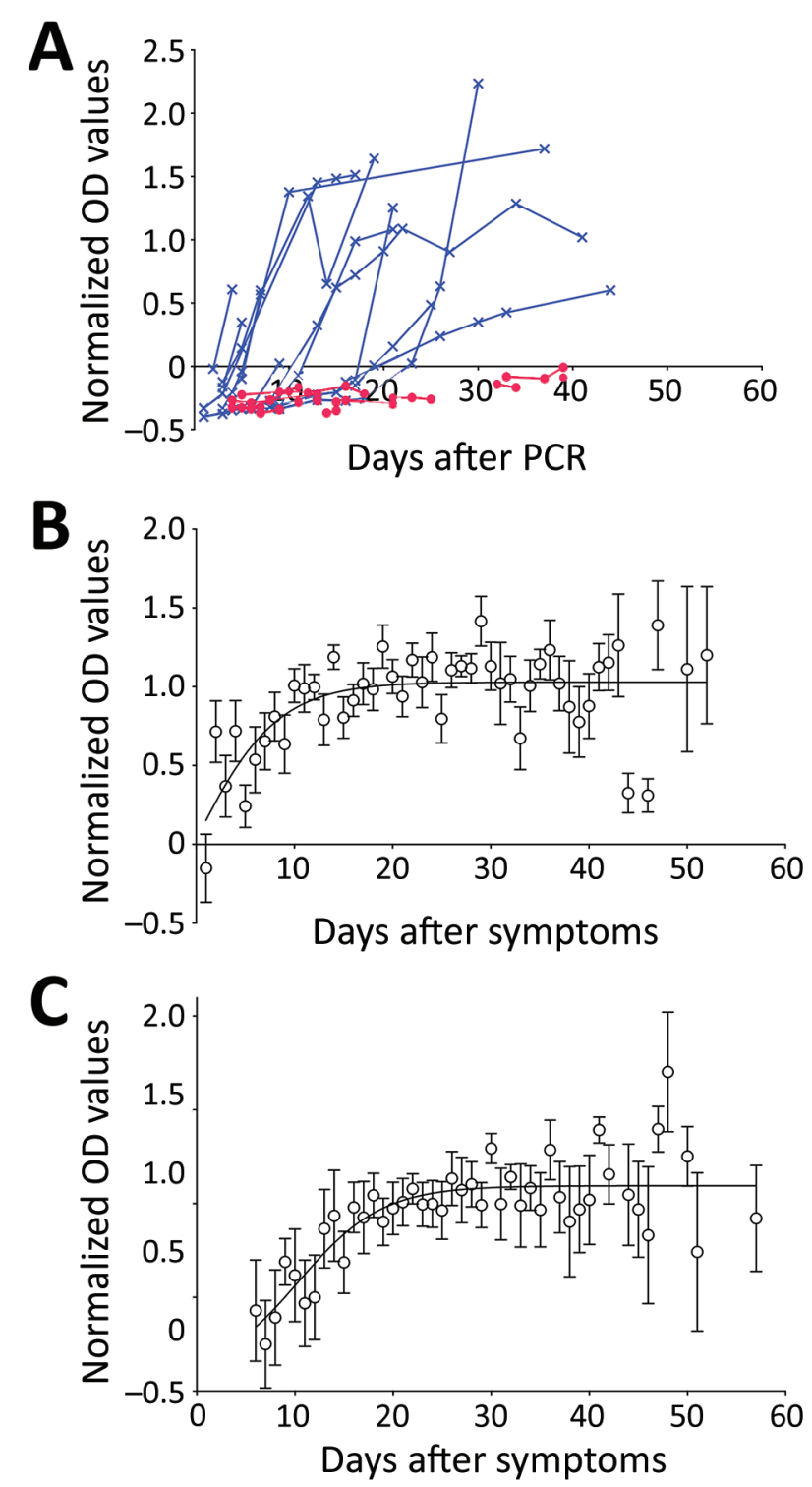

Figure 1. Antibody dynamics in patients with severe acute respiratory syndrome coronavirus 2, United Kingdom, 2020. A) NOD by days after first positive PCR result. Blue indicates seroconverting patients; red indicates nonseroconverting patients. B) Mean ( \pm SEM) NODs ( $\geq 3$ samples per time point; $n=48$ ) by days after first positive PCR result for those who seroconverted. A 4-parameter sigmoidal unconstrained model is shown $\left(r^{2}=0.45\right)$. C) Mean ( \pm SEM) NODs ( $\geq 3$ per time point; $n=45$ ) by days after symptom onset for patients who seroconverted. A 4-parameter sigmoidal unconstrained model is shown $\left(r^{2}=0.63\right)$. NOD, normalized optical density. 

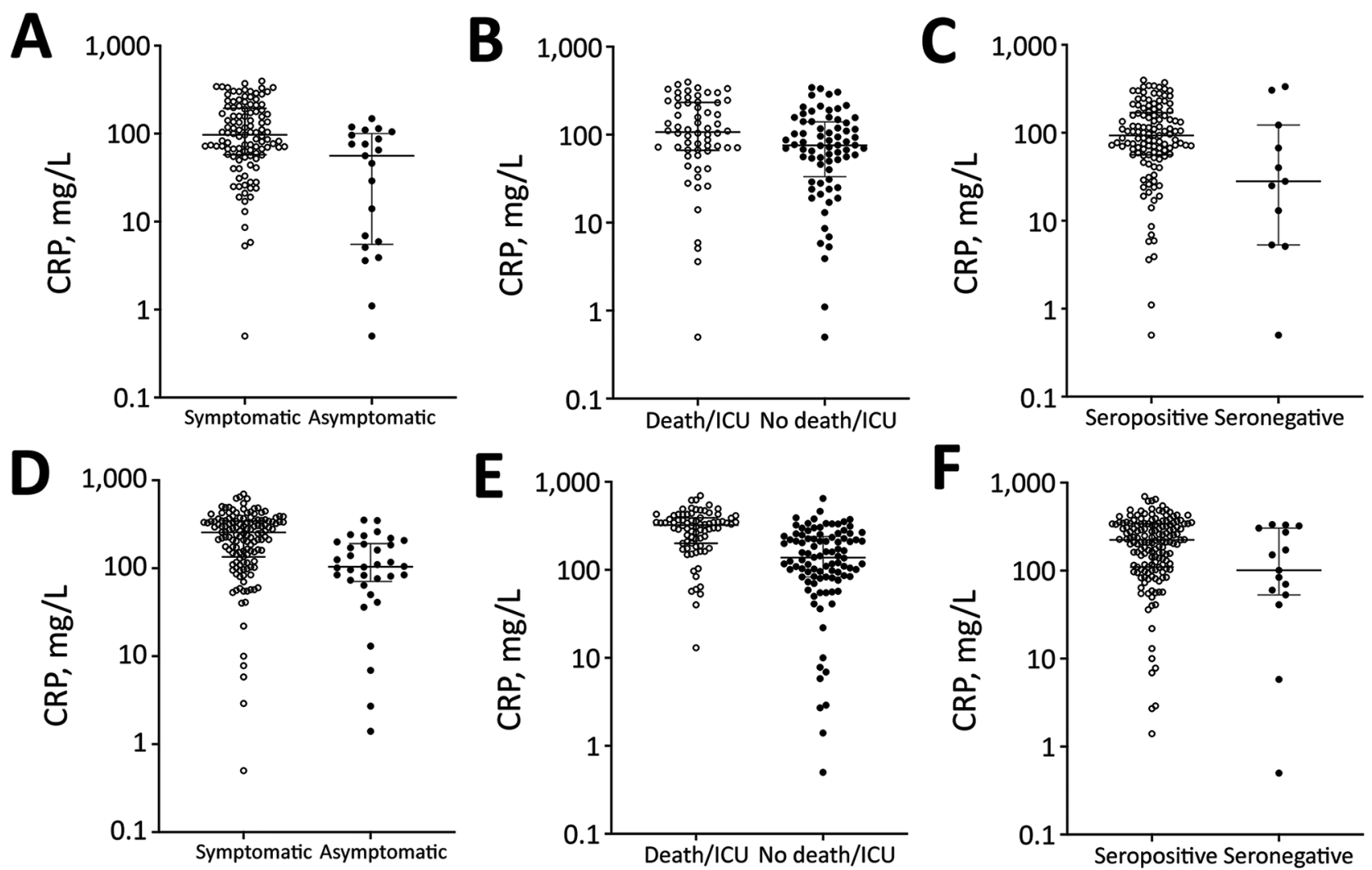

Figure 2. Relationships between CRP levels, symptoms, outcomes, and NODs of patients with severe acute respiratory syndrome coronavirus 2, United Kingdom, 2020. A-C) CRPs at diagnosis for A) 113 symptomatic (open circles) and 21 asymptomatic (closed circles) patients (CRP 97 vs. 56; $p<0.01$ ); B) 62 patients admitted to intensive care and/or who died (open circles) and 72 who were not admitted to intensive care (closed circles) (CRP 107 vs. 75.5; $p=0.01$ ); C) 123 patients who seroconverted (open circles) and 11 who did not (closed circles) (CRP 93 vs. 28; $p=0.04)$. D-F) Peak CRPs corresponding to the populations in A-C: $D) 255(n=142)$ vs. 104 ( $n$ $=34)(p<0.01)$; E) $322(n=80)$ vs. $137.5(n=96)(p<0.01) ; F) 224(n=161)$ vs. $101(n=15)(p=0.03)$. Statistical significance calculated using Mann-Whitney test for CRPs (mg/L). CRP, C-reactive protein; NOD, normalized optical density.

was relatively rapid; NODs remained stable for weeks after infection (Figure 1). The probability of seroconversion was associated with increased age and concurrent conditions such as hypertension and increased body mass index. Higher NODs were associated with nonWhite race, admission to hospital, and higher peaks for inflammatory markers, such as CRP. Higher antibody titers are associated with clinical severity (i.e., death or admission to intensive care during study) in our cohort, in agreement with findings from other studies (4).

CRP is a sensitive marker of elevated proinflammatory cytokines, including interleukin 6. These cytokines might play a central role in cytokine release syndrome, which is associated with increased risk for death (Y. Woo, unpub. data, https://osf.io/ mxsvw). Interventions such as tocilizumab, an interleukin-6 receptor antibody, interrupt the proinflammatory cascade. Such interventions might limit disease progression and reduce risk for death (9; E. Baker, unpub. data, https://osf.io/d2nh8); they are being studied in several randomized clinical trials. In our study, a small proportion of patients did not seroconvert within 20 days after testing positive for SARS-CoV-2 infection. Several mechanisms might explain this finding. First, these patients might never seroconvert. Second, their immune responses might be confined to other antigens or mediated through $\mathrm{T}$ cells. Another probable explanation is that some relatively mild infections might be restricted to the mucosal cells of the respiratory tract, where antibody responses are dominated by the secretory immune system. In this scenario, the systemic immune system might produce little or no IgG.

The association of higher NODs with elevated CRPs could indicate several potential pathways. For example, antibody responses might be closely related to cytokine response syndrome, which in turn is associated with more severe disease and death. Alternatively, elevated CRPs might indicate a more pronounced innate immune response in persons already 
Table 2. Selected laboratory values of patients with severe acute respiratory syndrome coronavirus 2 infection, United Kingdom, $2020^{*}$

\begin{tabular}{|c|c|c|c|c|}
\hline \multirow[b]{2}{*}{ Variable (reference range) } & \multicolumn{2}{|c|}{ At diagnosis } & \multicolumn{2}{|c|}{ Peak } \\
\hline & No. & Median (IQR) & No. & Median (IQR) \\
\hline C-reactive protein (0-5 mg/L) & 134 & $86(52.5-164)$ & 176 & $215.5(103-334)$ \\
\hline Nadir lymphocytes count $\left(1.1-4.0 \times 10^{9} / \mathrm{L}\right)$ & 134 & $0.9(0.6-1.4)$ & 177 & $0.6(0.4-0.8)$ \\
\hline Ferritin $(30-400 \mu \mathrm{g} / \mathrm{L})$ & 42 & $1,084(630-1,721)$ & 89 & $1,335(846-2,758)$ \\
\hline Fibrinogen $(1.6-4.8 \mathrm{~g} / \mathrm{L})$ & 133 & $5.5(4.2-6.7)$ & 166 & $7.0(5.5-9.15)$ \\
\hline D-dimer (21-300 ng/mL) & 64 & $704(395-1,079)$ & 111 & $1,905(498-4,095)$ \\
\hline Lactate dehydrogenase $(0-250 \mathrm{U} / \mathrm{L})$ & 47 & $475(280-597.5)$ & 87 & $490(344.5-704)$ \\
\hline
\end{tabular}

at risk for severe disease and death. This heightened innate response might be associated with a higher viral load (potentially caused by enhanced viral replication mechanisms) and genetic interactions that influence innate inflammatory pathways. Therefore, a higher viral load might lead to higher NODs for antibodies in the acquired immune response pathways. Small trials on the potential therapeutic benefits of interventions using passive antibody transfer (10) suggest that the heightened innate response hypothesis is more probable (11). Higher antibody responses are also associated with higher doses of a nonreplicating Ad5-vectored vaccine for SARS-CoV-2 (12).

Limitations of PCR include difficulties with sampling; different sample types and techniques yield varying results. Furthermore, PCR demonstrates diminishing diagnostic yield for COVID-19 as respiratory viral loads fall and symptoms subside $(8,13)$. It might also produce false positives caused by lingering viral nucleic acid, which is not infective yet can persist for weeks after infection. Contamination could also occur during sample handling; because PCR requires amplification steps, this assay has heightened risk for contamination. Serologic testing, and the ability to detect viral antigens, may increase diagnostic accuracy for COVID-19. Our findings support early studies suggesting that physicians should consider these diagnostic modalities in conjunction, especially when a patient has negative PCR results but has symptoms of COVID-19 (6). Many COVID-19 patients experience a delay in care, a trend that emphasizes the importance of containment strategies that encourage isolation.

One limitation of our study is that it is based mainly on hospitalized patients, of whom 1 in 5 did not have COVID-19 symptoms. Further studies should document antibody dynamics of patients with less severe infections, such as healthcare workers (14), and patients with low viral loads at the time of consultation. Our findings will complement the large cross-sectional and longitudinal serologic surveys, especially as highquality tests become more widely available. NODs were within a limited dynamic range (we could not conduct dilution studies because of small sample volumes) but nevertheless associated with clinically relevant features of COVID-19. Prospective studies are assessing the relationships between viral loads and serologic responses in patients. Regular and long-term serologic assays will be essential to monitoring the duration of the humoral response and its protective role against SARS-CoV-2.

When interpreting serologic assays of COVID-19 patients, physicians should consider factors that can influence the probability of seroconversion. Our study elucidates some of these factors. We found that less severe infections and younger age were associated with reduced probability of seroconversion. Risk factors for more severe disease, such as nonWhite race, increased age, and hypertension, are also associated with increased inflammatory responses, higher normalized antibody titers, and probability of seroconversion.

This article was preprinted at https:/ / www.medrxiv.org/ content/10.1101/2020.06.07.20124636v2.

\section{Acknowledgments}

We are greatly indebted to the National Association of Blood Bikes for their unwavering assistance in transporting samples, prototypes, and validated devices between Liverpool, Bedford, and London. Without their help, we could not have delivered 2 Conformité Européenne-marked products in 8 weeks from project launch. We also thank the tireless laboratory staff and informatics team, especially James Lawrence, of South West London Pathology.

This study was supported by a UK Department for International Development/Wellcome Trust Epidemic Preparedness coronavirus grant (grant no. 220764/Z/20/Z) to J.R.A.F., S.K., H.M.S., E.R.A., L.E.C., A.A.S. and by the Rosetrees Trust and the John Black Charitable Foundation (grant no. M959) to H.M.S., D.J.C., T.P., S.K., and J.R.A.F. In addition, D.E.K. is supported by Medical Research Council, UK, under fellowship no. MR/P019978/2. H.M.S. is supported by the Wellcome Trust Institutional Strategic Support Fund (204809/Z/16/Z) awarded to St George's, University of London, London. 


\section{About the Author}

Dr. Staines leads a research group at St George's, University of London, London. His main research interests are infectious diseases and diagnostics.

\section{References}

1. Yang X, Yu Y, Xu J, Shu H, Xia J, Liu H, et al. Clinical course and outcomes of critically ill patients with SARS-CoV-2 pneumonia in Wuhan, China: a single-centered, retrospective, observational study. [Erratum in: Lancet Respir Med. 2020;8:e26]. Lancet Respir Med. 2020;8:475-81. https:/ / doi.org/10.1016/S2213-2600(20)30079-5

2. Chan JF, Yuan S, Kok KH, To KK, Chu H, Yang J, et al. A familial cluster of pneumonia associated with the 2019 novel coronavirus indicating person-to-person transmission: a study of a family cluster. Lancet. 2020;395:514-23. https:/ / doi.org/10.1016/S0140-6736(20)30154-9

3. Our World in Data. Coronavirus pandemic (COVID-19). 2020 [cited 2020 Jun 5]. https:/ / ourworldindata.org/coronavirus

4. Zhao J, Yuan Q, Wang H, Liu W, Liao X, Su Y, et al. Antibody responses to SARS-CoV-2 in patients of novel coronavirus disease 2019. Clin Infect Dis. 2020 Mar 28 [Epub ahead of print]. https://doi.org/10.1093/cid/ciaa344

5. Ruan Q, Yang K, Wang W, Jiang L, Song J. Clinical predictors of mortality due to COVID-19 based on an analysis of data of 150 patients from Wuhan, China. [Erratum in: Intensive Care Med. 2020;46:1294-7]. Intensive Care Med. 2020;46:846-8. https:/ / doi.org/10.1007/ s00134-020-05991-x

6. Guo L, Ren L, Yang S, Xiao M, Chang D, Yang F, et al. Profiling early humoral response to diagnose novel coronavirus disease (COVID-19). Clin Infect Dis. 2020;71:77885. https://doi.org/10.1093/cid/ciaa310

7. Kucirka LM, Lauer SA, Laeyendecker O, Boon D, Lessler J. Variation in false-negative rate of reverse transcriptase polymerase chain reaction-based SARS-CoV-2 tests by time since exposure. Ann Intern Med. 2020;173:2627. https:// doi.org/10.7326/M20-1495
8. Long QX, Liu BZ, Deng HJ, Wu GC, Deng K, Chen YK, et al. Antibody responses to SARS-CoV-2 in patients with COVID-19. Nat Med. 2020;26:845-8. https:// doi.org/ 10.1038/s41591-020-0897-1

9. Xu X, Han M, Li T, Sun W, Wang D, Fu B, et al. Effective treatment of severe COVID-19 patients with tocilizumab. Proc Natl Acad Sci U S A. 2020;117:10970-5. https:/ / doi.org/ $10.1073 /$ pnas. 2005615117

10. Salazar E, Perez KK, Ashraf M, Chen J, Castillo B, Christensen PA, et al. Treatment of coronavirus disease (COVID-19) patients with convalescent plasma. Am J Pathol. 2020;190:1680-90. https:/ / doi.org/10.1016/ j.ajpath.2020.05.014

11. Jiang S, Hillyer C, Du L. Neutralizing antibodies against SARS-CoV-2 and other human coronaviruses. [Erratum in: Trends Immunol. 2020;41:545]. Trends Immunol. 2020;41:355-9. https:// doi.org/10.1016/j.it.2020.03.007

12. Zhu FC, Li YH, Guan XH, Hou LH, Wang WJ, Li JX, et al. Safety, tolerability, and immunogenicity of a recombinant adenovirus type- 5 vectored COVID-19 vaccine: a doseescalation, open-label, non-randomised, first-in-human trial. Lancet. 2020;395:1845-54. https:/ / doi.org/10.1016/ S0140-6736(20)31208-3

13. Xie C, Jiang L, Huang G, Pu H, Gong B, Lin H, et al. Comparison of different samples for 2019 novel coronavirus detection by nucleic acid amplification tests. Int J Infect Dis. 2020;93:264-7. https:// doi.org/10.1016/j.ijid.2020.02.050

14. Fafi-Kremer S, Bruel T, Madec Y, Grant R, Tondeur L, Grzelak L, et al. Serologic responses to SARS-CoV-2 infection among hospital staff with mild disease in eastern France. EBioMedicine. 2020;59:102915. https:// doi.org/10.1016/ j.ebiom.2020.102915

Address for correspondence: Sanjeev Krishna, Institute for Infection \& Immunity, St. George's, University of London, Cranmer Terrace London, SW17 0RE, UK; email: s.krishna@sgul. ac.uk; Tim Planche, Department of Medical Microbiology, St. George's University Hospitals NHS Foundation Trust, Blackshaw Rd, London, SW17 0QT, UK; email: tplanche@sgul.ac.uk 